\title{
Enabling Low-dose Liquid-phase TEM with Advanced Signal Processing, Machine Learning, and Molecular Simulation
}

\author{
John Smith ${ }^{1}$ and Qian Chen ${ }^{2}$ \\ ${ }^{1}$ University of Illinois at Urbana-Champaign, United States, ${ }^{2}$ University of Illinois at Urbana-Champaign, \\ Urbana, Illinois, United States
}

Both the structure and dynamics of biomacromolecules - put together, the source of their functionalityare bestowed upon them by the properties of their ever-fluctuating, liquid environment. In particular, water mediates the inter- and intramolecular forces that give proteins, nucleic acids, and macromolecular assemblies their structure, and it serves as a dynamic medium for the structural fluctuations required to execute physiological tasks like molecular locomotion or gene replication. There have been increasing efforts to harness liquid-phase transmission electron microscopy (TEM) - a real-time nanoscopic imaging technique - to study these and other biophysical phenomena with nanometer and sub-second resolution [1], as this combination of spatial and temporal resolution is challenging to achieve with other experimental tools. However, the fragility of organic materials under electron illumination makes it imperative to use low electron dose rates when imaging such specimens, to avoid structural damage and experimental artifacts [2,3]. Combined with the already limited intrinsic visibility of biomolecules in an electron microscope and the presence of relatively thick liquid layers, images or "movies" produced by liquid-phase TEM are often confounded by extremely low contrast and signal-to-noise, which introduce fundamental challenges in data interpretation.

Here we will present an integration of concepts from advanced signal processing [4], machine learning [5], electron imaging theory [6], and molecular simulation which, together or independently, aid in improving signal-to-noise and interpretability in low dose liquid-phase electron microscopy movies (Figure 1). Some case studies in real liquid-phase TEM data from (bio)macromolecular specimens will be used to illustrate the structural, physical, and thermodynamic information which can be garnered in the process. First among these are signal processing methods that are focused in temporal, rather than spatial variations in image intensity. It is common to perform image filtering in the spatial ( $x y)$ domain, but straightforward methods like a Gaussian or median filter can lower the image resolution and are less beneficial in images with high levels of noise. However, algorithms that operate on the temporal intensity fluctuations present in movies - borrowed from concepts used in aerospace navigation and tracking - are a complementary way to reduce the effects of imaging or detector noise without affecting the spatial content. Second, we will present a machine learning method—based on compressive sensing [5] - also capable of improving the signal-to-noise by "learning" a denoised representation directly from experimental data. Finally we will demonstrate how, through a fusion of molecular dynamics (MD) simulation and electron contrast transfer theory, contrast variations in otherwise difficult-to-interpret images can be used to infer molecular-level structure variation. In particular, with principles from electron optics, a large library of equilibrium structures generated by MD simulations can be used as a "reference manual" for contrast variation in experimental images and, in some cases, used to identify key structural features, providing additional and sometimes higher resolution morphological information. 

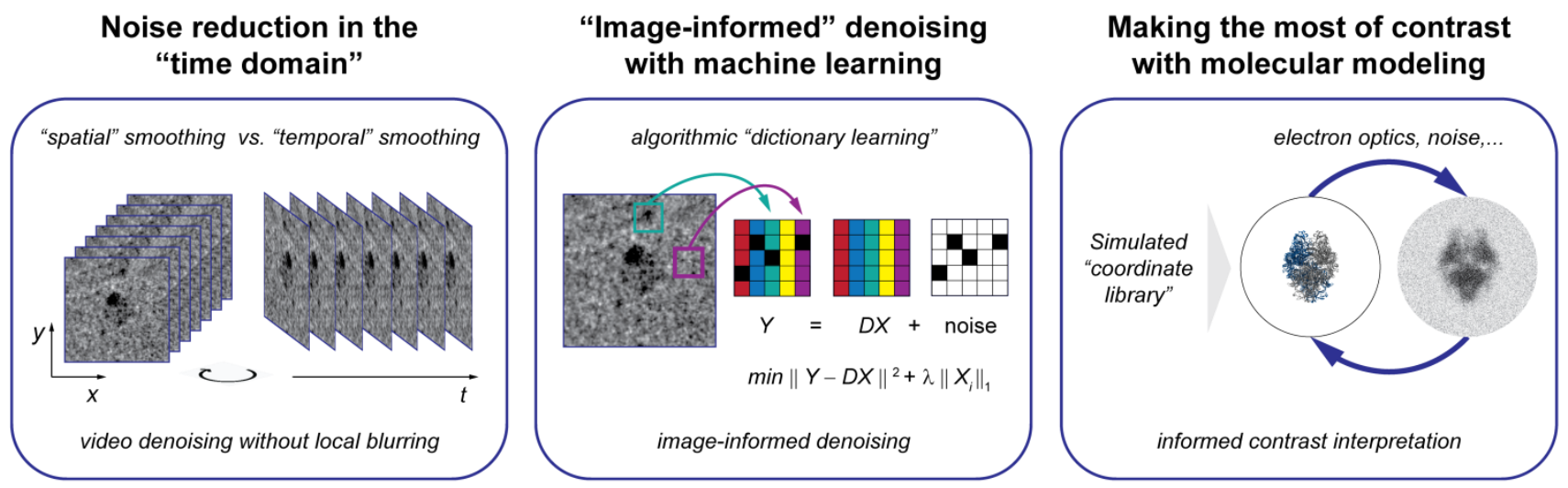

Figure 1 Low imaging contrast and signal-to-noise in low-dose liquid-phase electron microscopy data can be combated with a variety of data-analytical approaches. Instead of spatial filtering, algorithmic enhancement in the time domain present in "movies" offers a potent and complementary means of denoising, as do machine-learning based denoising methods. Insight can also be provided by predicting "possible" images on the basis of MD simulations and electron optical theory.

\section{References}

[1] JW Smith and Q Chen, J. Mater Chem. B 8 (2020), p. 8490.

[2] NM Schneider et al., J. Phys. Chem. C 118 (2014), p. 22373.

[3] J Kim et al., ACS Nano 10 (2016), p. 9801.

[4] RE Kalman, J. Basic Eng. 82 (1960), p. 35.

[5] A Stevens et al., Microscopy 63 (2013), p. 41.

[6] H Rullgård et al. J. Microsc. 243 (2011), p. 234.

[7] The authors acknowledge funding from the Air Force Office of Scientific Research (AFOSR FA955020-1-0257) 